\title{
La formación del documentalista y archivista en documentación audiovisual en Costa Rica.
}

\section{Xinia Rojas González}

\section{Recibido: 24 de agosto de 2015}

\section{Aceptado: 10 de septiembre de 2015}

\section{Resumen}

Se analizan los planes de estudios conducentes a la obtención de los títulos universitarios en archivología y bibliotecología en Costa Rica con el fin de identificar la formación relacionada con la documentación audiovisual. Para ello se han analizado los planes de estudios de las siguientes Escuelas: Escuela de Bibliotecología y Ciencias de la Información de la Universidad de Costa Rica (EBCl), Escuela de Bibliotecología, Documentación e Información de la Universidad Nacional (EBDI), Escuela de Ciencias Sociales y Humanidades de la Universidad Estatal a Distancia, la Escuela de Bibliotecología de la Universidad Autónoma de Centroamérica y la Escuela de Historia y Archivística de la Universidad de Costa Rica. Se desarrolla igualmente un estado de la cuestión revisando la literatura sobre estudios previos sobre planes de estudios y sobre la importancia de la documentación audiovisual.

\section{Pallabras clave}

Documentación audiovisual, Formación universitaria, planes de estudios, Costa Rica, Archivología, Bibliotecología

\section{The educational framework around audiovisual documentation in Costa Rica}

\section{Abstract}

Study plans required to obtaining university degrees in Archival and Library Sciences in Costa Rica are analyzed with the purpose of identifying the educational framework around audiovisual documentation. To achieve this objective, study plans from the following schools have been analyzed: Faculty of Information Library Sciences of the Costa Rica University, Faculty of Library, Documentation and Information of the National University, Faculty of Social Sciences and Humanity of the Universidad Estatal a Distancia, Faculty of Library of the University Autonoma of Centroamerica and the Faculty of History and Archival Sciences of the Costa Rica University. Additionally, a status analysis is developed by reviewing existing documentation on previous study plans research works and also about the important of audiovisual documentation.

\section{Keywords}

Audiovisual document, University educación, formation university, study planes, Costa Rica, Archivist, Librarianship

http://dx.doi.org/10.5209/rev CDMU.2015.v26.50632 


\section{INTRODUCCIÓN}

En la actualidad el fondo documental de las distintas unidades de información esta compuesto por una gran riqueza de formatos en donde se registra la información. Estos formatos pueden ser: sonoros, textuales, fotográficas y-o audiovisual. Esta condición exige que la formación del documentalista de información y el archivista debe de estar acorde con las tecnologías que ofrece el mercado mundial, así como conocimiento en los distintos formatos donde se registra esta información y las demandas de los usuarios que requieren información. Por ello, se hace necesario valorar los planes de estudio y los cursos que reciben los profesionales, las distintas escuelas en donde se imparte las carreras en Costa Rica, con el fin de poder llegar a nuestro objetivo, que es precisamente, conocer la preparación académica para poder gestionar la documentación audiovisual.

Si bien es cierto, la mayoría de los bibliotecólogos y archivistas en este país pueden desempeñarse en cualquier de los tipos de unidades de información y archivos; existe una cantidad de secciones dentro de éstas, en donde se localizan fondos de información en soportes audiovisuales. No obstante, estos fondos deberían ser tratados, gestionados y conservados por un documentalista audiovisual, puesto que se pudo constatar que no se cuenta en el país con especialistas, sino existen conocimientos generales y la buena voluntad de los colegas que se desempeñan en estas bibliotecas y secciones.

Analizando los currículos de cada una de las disciplinas estudiadas se puede constatar que se recibe una formación muy generalizado, ejemplo de ellos son los cursos donde se estudia la descripción bibliográfica o archivística y la gestión de colecciones (documentales).

Como es lógico el problema repercute en algunas unidades de información en donde algunos de estos profesionales se desempeñan, puesto que es allí donde se hace necesaria contar con una especialización, como sería la documentación audiovisual.

\section{METODOLOGÍA}

Para la elaboración de este artículo fue necesario revisar la oferta curricular que ofrece cada una de las siguientes instituciones de educación superior: Bibliotecología y Ciencias de la Información de la Universidad de Costa Rica (EBCl), Escuela de Bibliotecología, Documentación e Información de la Universidad Nacional (EBDI), Escuela de Ciencias Sociales y Humanidades de la Universidad Estatal a Distancia, la Escuela de Bibliotecología de la Universidad Autonóma de Centroamérica y la Escuela de Historia y Archivística de la Universidad de Costa Rica.

Para realizar el análisis de la información recabada en el proceso de esta investigación se utilizó la información que aparece registrada en los programas de los cursos, los mismos se convirtieron en fuentes primarias fundamentales, ya que en ellas se plasman los contenidos y las actividades que los profesores realizan en el período de desarrollo del curso. En este proceso se realizaron visitas personales en la mayoría de las las instituciones antes señaladas, esta actividad permitió indagar con las directoras de las carreras, los cursos pertinentes a esta especialización. En otros de los casos, se consultó directamente al profesor del curso los contenidos que se estudian en los cursos. 


\section{LA FORMACIÓN PROFESIONAL EN CIENCIAS DE LA INFORMACIÓn}

La formación profesional es una actividad que busca desarrollar aptitudes en los individuos que les permitan alcanzar una vida productiva y satisfactoria. Cassanova (2003) afirma que la formación profesional es simultáneamente tres cosas: "Es una actividad de tipo educativo, es una actividad vinculada a los procesos de transferencia, innovación y desarrollo de tecnología y es un hecho laboral” (p.10).

En cuanto a la actividad educativa -que es lo que el tema de interés de este artículo- Cassanova menciona que:

"se orienta a proporcionar los conocimientos, habilidades y destrezas necesarios para desempeñarse en el mercado de trabajo, sea en un puesto determinado, una ocupación o un área profesional. Actúa a su vez de forma complementaria a las otras formas de educación, formando a las personas no sólo como trabajadores sino también como ciudadanos" (p.10)

La formación del profesional en la disciplina bibliotecológica y archivística, así como sus planes de estudios han sido estudiados principalmente por docentes que laboran en distintas escuelas y facultades, tal es el caso de Pirela (2008), Tejada (2008), Escalona (2008), Muñera (2010) y Vallejo (2011), quienes en relación a la formación coinciden en la necesidad de estar valorando los programas de estudio y las escuelas de enseñanza, así como la importancia de actualizarlos periódicamente.

La formación bibliotecológica costarricense estudiada por Rodríguez Salas (2008) determina las habilidades y las competencias que poseen los estudiantes que concluyen estudios en el plan de Bachillerato en Bibliotecología y Documentación de la Escuela de Bibliotecología, Documentación e Información de la Universidad Nacional de Costa Rica. El este estudio se visualiza los conocimientos las habilidades, destrezas y actitudes apropiadas que se consideran para la elaboración de los perfiles profesionales de la institución. Y más recientemente Bustamante Mora, Cascante Ardón, Corderon Mélendez, Rodríguez Vindas, Saavedra Arias y Zúñiga Villalobos (2014) desarrollaron un perfil ocupacional del profesional de graduado en Ciencias de la Información por parte de la Escuela de Bibliotecología y Ciencias de la Información de la Universidad de Costa Rica, donde se destacan también los conocimientos, habilidades y actitudes. Para su elaboración se contrastó el perfil de salida y la formación ofrecida por la carrera.

Un estudio similar realiza Echavarría (2002) en la archivística costarricense. La autora analiza las tendencias de las nuevas organizaciones, el ambiente laboral y el profesional que demandan la sociedad costarricense y las respuestas que brindan las universidades, que deriva en una propuesta actualizada del plan de estudios.

La formación del documentalista costarricense -al igual que en el resto de los países- nace precisamente de la necesidad que se deriva de la sociedad de contar con un profesional en este campo. Es así como la Escuela de Bibliotecología y Ciencias de la Información de la Universidad de Costa Rica (EBCI) logra posicionarse históricamente como líder en la enseñanza de la Bibliotecología.

Con los primeros graduados se atendían las distintas bibliotecas que para ese tiempo ya existían en la sociedad costarricense. Posteriormente con el desarrollo de las tecnologías, nacen las bibliotecas especializadas y 
Centros de Documentación, que por consiguiente requerían contar con un documentalista habilidoso en búsquedas, con conocimientos en la gestión de la información. Esta demanda, da pie a que la $\mathrm{EBCl}$ deba de abrir la carrera especializada en Ciencias de la Información. Es así como desde 1989 y hasta la fecha la Escuela de Bibliotecología y Ciencias de la Información (EBCl) imparte esta carrera.

En el año 1977, la Universidad Nacional de Costa Rica (UNA) ofrece un diplomado en Bibliotecología con una duración de aproximadamente 2 años y medio, que brinda la posibilidad a los estudiantes que ingresan en esta carrera especializarse en documentación. A partir de 1986 ofrece la titulación de bachillerato.

En 1990, la Universidad Estatal a Distancia (UNED) ofrece a la población costarricense la carrera con una titulación en Bachillerato en Bibliotecología y Nuevas Tecnologías de Información y Comunicación.

Actualmente, la única Universidad privada que desde el año 1990 imparte la carrera de Bibliotecologia es la Universidad Autónoma de Centroamérica con un titulo de grado de Bachillerato en Bibliotecología.

Sobre la formación en archivística Echevarría Solís y Rivas Fernández (2006) señalan:

"La Carrera de Archivística se imparte en la Escuela de Historia de la Universidad de Costa Rica se inició en el año de 1978, con un plan de estudios a nivel de pre-grado que otorgaba el título de Diplomado, formando un técnico medio que asumiera los procesos necesarios para el tratamiento de los documentos especialmente en un archivo final, dada la estrecha relación entre la historia y los archivos. Posteriormente, en el año de 1992 se aprobó el plan de estudios de Bachillerato que conservaba como salida lateral al Diplomado, en respuesta a las demandas que planteaba la existencia de un Sistema Nacional de Archivos, aprobado según Ley 7202 del 24 de octubre de 1990, como marco jurídico para la protección y desarrollo de los archivos". (p.1).

\section{LA FORMACIÓN EN DOCUMENTACIÓN AUDIOVISUAL}

Como se puede apreciar el documentalista y archivista se forma principalmente en la universidad pública, con una plan de estudios general, pero no tiene una especialización en medios de comunicación, como si esta establecido en otros países, tal es caso de España que brinda la posibilidad a estos profesionales de optar por una titulación en esta área mediante el Máster impartido por la Universidad Carlos III de Madrid en colaboración Radio Televisión Española (RTVE). Si bien es cierto, en el país existen centros de documentación en canales de televisión, estos no son atendidos por profesionales puesto que tal y como señalan Royan y Cremer (2004) la formación del profesional que atiende estas unidades de información debe ser un especialista en materiales audiovisuales y multimedia y saber manejar los equipos respectivos.

La gestión documental en los medios de comunicación cuenta con diferentes vertientes, ya que en los medios se gestiona tanto información textual, sonora y audiovisual atendiendo al tipo de centro en el que se trabaja. Los tipos documentales serán diferentes si la labor se desarrolla para televisión, radio o prensa escrita. No obstante, existe una clara hibridación de medios de comunicación y de formatos con la presencia de los medios en red, generando confluencia de formatos y métodos de transmisión. 
Tradicionalmente, especialmente en España, han existido asignaturas ligadas a los medios de comunicación, especialmente por la relación directa que desde siempre ha tenido el Departamento de Biblioteconomía y Documentación con las Ciencias de la Información (así se denomina, aunque realmente debiera llamarse Ciencias de la Comunicación, como en el resto de países hispanohablantes) en la Universidad Complutense de Madrid.

La asignatura de Documentación periodística (también denominada como Documentación Informativa en alguno de los planes de estudio) ha estado presente en la práctica totalidad de los planes de estudios españoles, pero no así en los planes de estudios en los países de América Latina.

Igualmente se ha podido comprobar la existencia de asignaturas concretas sobre Documentación Audiovisual, Documentación textual para medios de comunicación, y Documentación Sonora especialmente orientada a los medios radiofónicos.

De igual manera, existen profesores y líneas de investigación que trabajan sobre medios de comunicación en sus diferentes vertientes, contando por tanto con una importante literatura científica sobre dicha temática. Valga indicar la existencia de investigadores provenientes tanto de la académica como de los medios de comunicación. Destacamos a autores y trabajos procedentes de la académica (Valle Gastaminza, 1999) (Caldera-Serrano, 2004) (López Yepes, 1992) así como de medios de comunicación (Lopéz de Quintana, 2000) (Hidalgo, 2005) (Afuera, 1998).

Igualmente encontramos revistas que centran su línea de trabajo en la gestión de la información en medios de comunicación tales como "Documentación de las Ciencias de la Información" (http://revistas.ucm.es/index.php/DCIN) y mucho más concretamente para información audiovisual "Cuadernos de Documentación Multimedia" (http://revistas.ucm.es/index.php/CDMU). Aunque en otras revistas existen publicaciones sobre dicha temática, destacando "Revista General de Información y Documentación" (http://revistas.ucm.es/index.php/RGID/), El Profesional de la Información (http://www.elprofesionaldelainformacion.com/index.html), e incluso pueden encontrar un nuevo relevante de trabajos en Investigación Bibliotecológica (http://iibi.unam.mx/revistaCuib.html).

Si nos centramos concretamente en la documentación audiovisual para medios de comunicación encontramos mucha relación entre trabajos de la Universidad y los medios de comunicación, lo que ha ayudado a la interrelación entre ambas realidades generando trabajos con la doble visión (Caldera-Arranz, 2012), trabajos que van desde lo más general y líneas de investigación centradas en el desarrollo tecnológico y el cambio de paradigma de trabajo del analógico a lo digital (Caldera ; Sánchez, 2007) (Pérez-Aguera et al. 2004).

Con ello queremos reseñar que existe una interrelación entre los investigadores, empresas audiovisuales y de medios de comunicación en general, que hacen posible y viable la presencia de asignaturas específicas para formar al documentalista audiovisual (tanto en televisión como en información cinematográfica). Pero la realidad en Costa Rica nos hace visualizar la falta de existencia de investigadores en dicha disciplina (no sólo en audiovisual sino en medios de comunicación) y la falta de relación e interés por las empresas televisivas por hacer públicos sus trabajos o interactuar con la Universidad. De ahí esta falta de relación y por tanto la inexistencia de asignaturas. 
Por lo tanto, por alguna parte de esta cadena debemos comenzar a provocar que la documentación audiovisual sea tenida en cuenta por parte de la Academia y por parte de la Empresa; por lo que parece importante que sea desde la formación y la investigación por la que comencemos a integrar este tipo documental en la formación y posteriormente y de manera profesional en las entidades televisivas.

\section{ANÁLISIS DEL PLAN DE ESTUDIOS DE LAS CARRERAS}

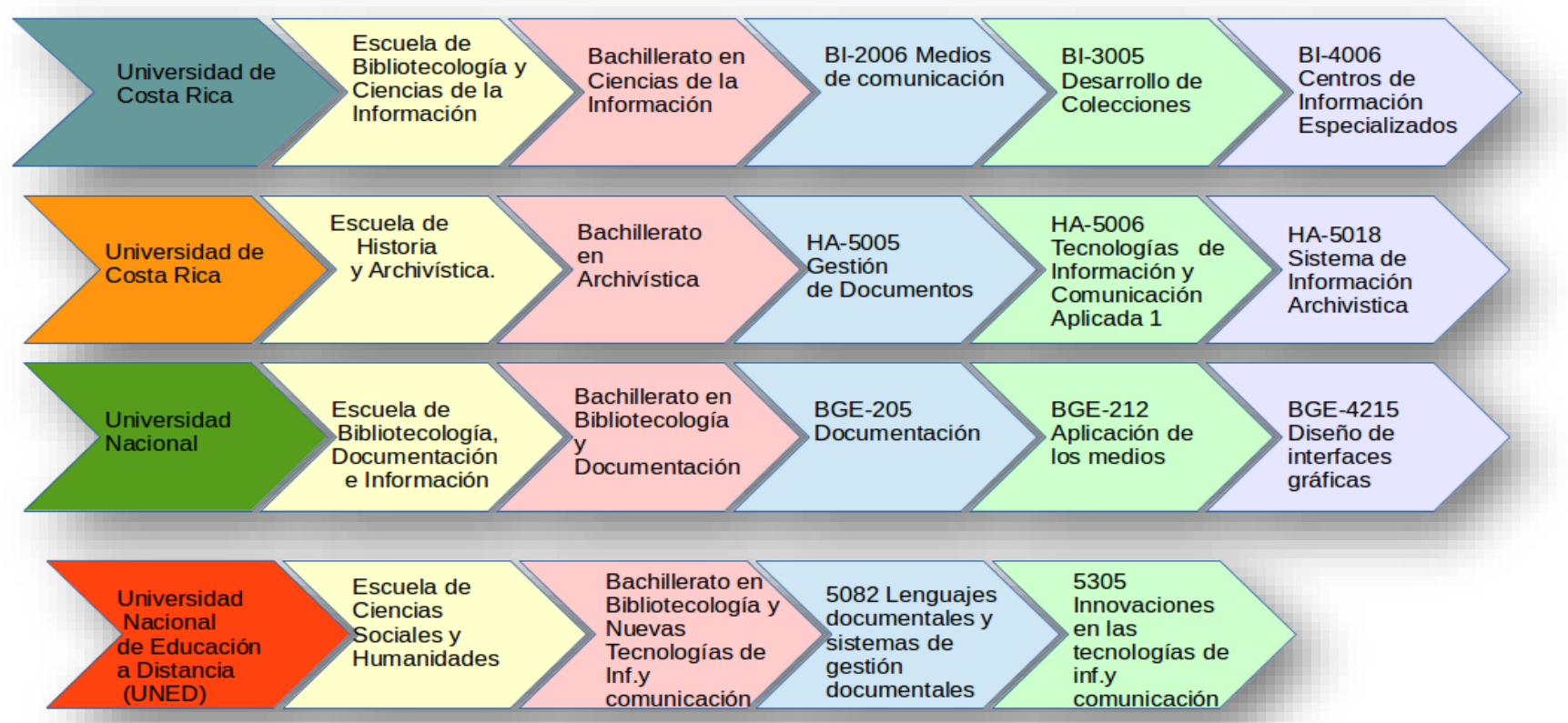

Figura 1. Universidades, titulación y cursos relacionados con documentación audiovisual

La Carrera de Documentación y Archivistica, como se puede apreciar en la figura 1, se ofrece a la sociedad costarricense principalmente a través de las universidades públicas. En el caso de la carrera de archivista, la Universidad de Costa Rica es la entidad que históricamente ha contado con una amplia trayectoria en el desarrollo de esta carrera. En la actualidad, se ha modificado el currículo educativo, resultado de ello, algunos 
contenidos han sido actualizados (HA-5005 Gestión de documentos y HA-5006 Tecnología de Información y Comunicación Aplicada 1 y otros aún no se han impartido, tal es caso del los curso HA5018 Sistemas de Información Archivistica que se tiene previsto para el año 2016).

La Escuela de Bibliotecología de la UACA no figura dentro del mismo, puesto que, a la hora de analizar la información, la Directora de la Carrera indicó solo contar con el curso de Procesamiento de la Información, curso donde se estudia las Reglas de Catalogación Angloamericanas. En este curso es donde el estudiante analiza los materiales audiovisuales para poder procesarlos técnicamente, pero solo se desarrolla el contenido en una Unidad del programa del curso. Esta misma situación se presenta en los demás cursos de procesos técnicos o procesamiento de la información (tienen distintas denominaciones según cada Universidad) donde por lo general tiene un nivel más alto.

En el caso de la Universidad Nacional dentro del plan de estudios solo se identificaron esos dos cursos, 082 Lenguajes documentales y sistemas de gestión documentales y 5305 Innovaciones en las Tecnologías de Información y Comunicación.

Es importante recalcar el papel que tiene el profesorado, puesto que no se evidencia haber recibido una formación en documentación audiovisual, lo que permite deducir que no existen especialistas en el país. Esta situación se convierte en una debilidad a la hora de impartir un curso, puesto que el profesorado carece de conocimiento y por ende del dominio sobre la temática.

En algunos de los casos, los profesores que imparten los cursos pertenecen a distintas disciplinas, pero al igual que pasan con los profesores de archivistica o bibiotecología tampoco son especialistas.

Figura 2. Cursos de la carrera de Bibliotecología y Ciencias de Información de la Universidad de Costa Rica relacionados con documentación audiovisual. 


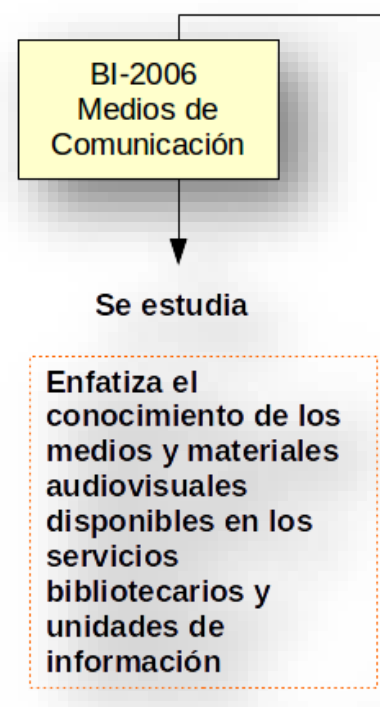

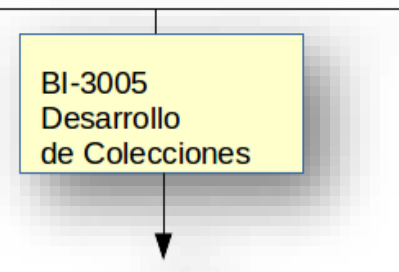

Se estudia

Principios básicos de los
procesos de selección;
adquisición; conservación;
restauración y evaluación
de colecciones, tanto
impresas romo
audiovisuales
electrónicas.

Relación con la documentación audiovisual
Unidad 1. Medios de comunicación e información escrita

Unidad 2. Medios de comunicación e información audiovisual y multimedial
Unidad 4. Evaluación de colecciones (publicaciones impresas como

electrónicas) con el fin de formar una colección en una Unidad de Información

\section{$\mathrm{Bl}-4006$}

Centros

de Información

especializados

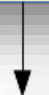

Se estudia

Brinda una visión general de la evolución de las ciencias de la información. Definir los aspectos más relevantes evaluar una colección así como su participación en redes y sistemas de información nacionales

\section{Universidad de}

Costa Rica.

Escuela de Bibliotecología y Cs. de la Información

Figura 2. Cursos de la carrera de Bibliotecología y Ciencias de Información de la Universidad de Costa Rica relacionados con documentación audiovisual

Fuente: Elaboración propia con los contenidos de los programas.

La figura 2, evidencia que el plan de estudios del Bachillerato en Bibliotecología y Ciencias de la Información de la Universidad de Costa Rica, que contiene 28 cursos, la documentación audiovisual solo se desarrolla en tres. En el caso de los cursos $\mathrm{Bl}-3005$ y Bl-4006, los estudiantes reciben un contenido, pero estrechamente ligada al desarrollo de colecciones y a Centros Especializados.

En el curso BI-2006 Medios de Comunicación (se encuentra dentro de los cursos de segundo año de la carrera) es donde más se analiza se analizan los medios de comunicación en dos de los contenidos del curso. Sin embargo, se podría considerar que para que un profesional dirija un Centro de Documentación especializado de una entidad televisiva, no contaría con la formación suficiente para desempeñarse de forma adecuada. 
Esta situación se refleja en los medios de comunicación televisivos, los cuales en su mayoría cuentan con un Centro de Documentación, pero los mismos no son dirigidos, ni por archivistas o bibliotecólogos.

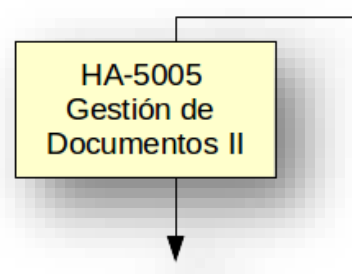

Se estudia

Introduce algunas
normas
internacionales
complementarias a
las ISO 15489 sobre
gestión de
documentos para su
posible aplicación y
uso en la
organización

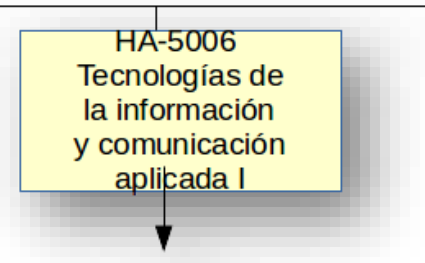

Se estudia

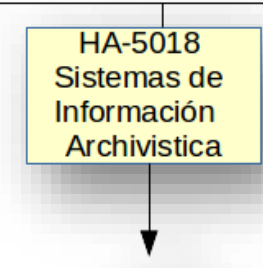

Se estudia
Universidad de

Costa Rica. Escuela de Historia.

Sección de

Archivistica

Relación con la documentación audiovisual

Unidad IV. Normas para la Preservación (UNE-ISOITR 15801:2014

Unidad V. Normas para Sistemas de Gestión de Documentos (Normas ISO16178-1, ISO 16172-2 y ISO16175-3)
Unidad III. El documento electrónico (gestión de documento electŕonico, características de los soportes no tradicionales, formatos para clases documentos electrónicos (texto, audio, sonoro)
Unidad VI. Gestión Electrónica de Documentos

Figura 3. Cursos de la carrera de Archivistica relacionados con la documentación audiovisual

Del análisis realizado en el plan de estudios de la Sección de Archivista de la Universidad de Costa Rica, se determina que al igual que la carrera de Bibliotecología, no existe un curso especializado en documentos audiovisuales, solo en los cursos que aparecen en la figura 3. Estos son los que al menos tiene un contenido o unidad didáctica que trata de forma general la temática.

En referencia al curso HA-5005 Gestión de Documentos II, este es el segundo curso que los estudiantes reciben, el primero de ellos brinda conocimientos básicos de lo que es la descripción de documentos, que sería el equivalente a los cursos de procesos técnicos que reciben los estudiantes de Bibliotecología. 


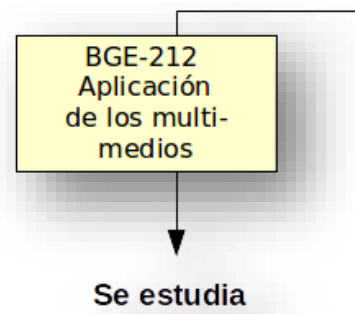

El curso pretende que el estudiante analice los procesos y técnicas para manipular y producir diferentes formas de multimedios y soportes documentales.

Unidad III. Creación de productos multimedia. (Tipos de formato de imagenes, vídeos, animaciones, sonidos, aplicaciones, sitios web, entre otros. Preproducción y postproducción y difusión

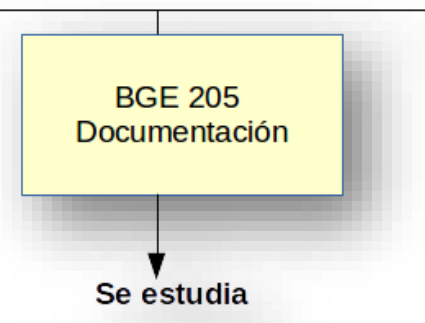

\begin{tabular}{lcc|}
$\begin{array}{l}\text { Estudia el ámbito de } \\
\text { disciplina }\end{array}$ & la \\
documentación. & & la
\end{tabular}

documentación.
BGE 4215

Diseño de inter-

faces gráficas

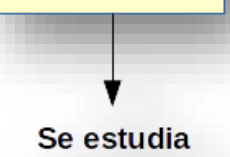

Se estudia procesos de comunicación global en formato digital, utilizando imágenes que integra sonido, vídeo, texto, fotografía diferentes medios tecnológicos.
Universidad

Nacional

de Costa Rica.

Escuela de Bibliotecología

y Documentación

Figura 4. Cursos de la carrera de Bibliotecología, Documentación de la Universidad Nacional de Costa Rica relacionados con documentación audiovisual

A diferencia con el plan de estudios de la carrera de Bibliotecología, los archivistas reciben un curso sobre preservación y en este curso se toma en cuenta la preservación de los los archivos audiovisuales.

Dentro de los cursos que ofrece la Escuela de Bibliotecología Documentación de la Universidad Nacional de Costa Rica, se destaca el curso BGE-212 Aplicación de los multimedios, el mismo tiene un componente práctico "que consiste en el diseño de un proyecto con formato informático del entorno multimedia (audio, imágenes, vídeo, texto, gráfico) con características y contenidos multimediales". (p.1). Además, los estudiantes deben de indagar una necesidad presente en una unidad de información documental y elaborar un proyecto. Este curso es el único en que los estudiantes analizan características y diferencias en relación a los formatos impresos. 
En el caso particular del curso BGE-205 Documentación, el curso esta orientado al diseño, organización, administración, recursos, manejo automatizado y evaluación de un centro de documentación que permite que el estudiante pueda trabajar el ciclo documental. Sin embargo, en el curso solo se estudia la relación con la documentación audiovisual en el contenido correspondiente a centros documentales especializados, tales como los audiovisuales.

Los cursos BGE 4215 y BGE 212, están relacionadas con el curso de BGE-212 Aplicación de los multimedios, ya que son cursos donde el estudiante debe de apoyarse con las tecnologías, de hecho, los profesores que imparten estos cursos son profesionales del área de las Tecnologías de Información.

En general, dentro del plan de estudio que ofrece la Universidad Nacional no se encontraron cursos especializados en documentación audiovisual.

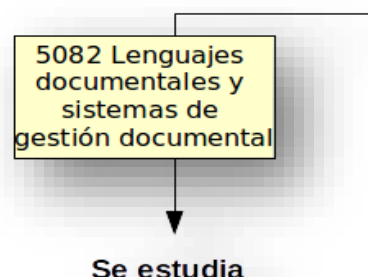

Se estudia

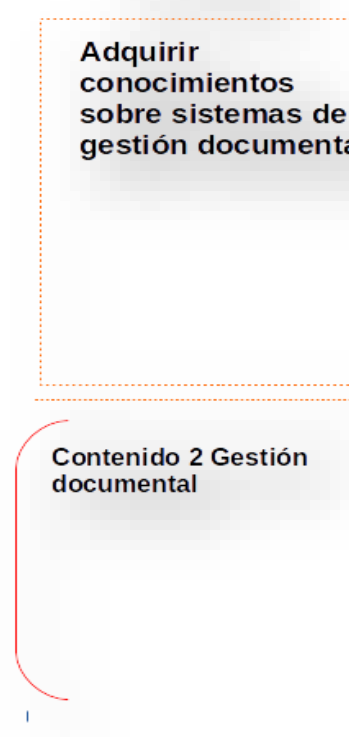

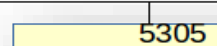

Innovaciones en las

tecnologías de la

Información y

comunicación

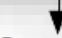

Se estudia

Analizar las tendencias y

propuestas de las TIC para

la propuesta de cambios y

actualizaciones

tecnológicas

Relación con la documentación audiovisual

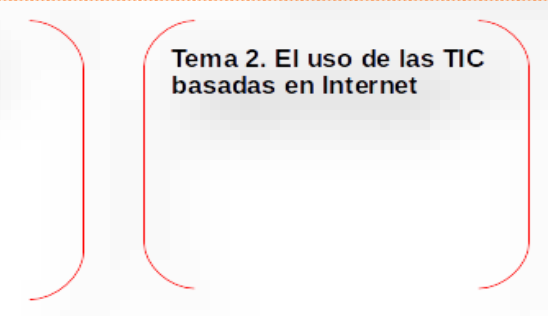

Figura 5. Cursos de la carrera de Bibliotecología Nuevas Tecnologías de Información y Comunicación de la Universidad Estatal a Distancia de Costa Rica relacionados con documentación audiovisual 
La propuesta curricular que ofrece la UNED se logra visualizar los cursos 5082 Lenguajes documentales y sistemas de gestión documental y 5305 Innovaciones en las tecnología de información y comunicación.

Al igual que las Escuelas de formación analizadas en las demás figuras también solo se ve un contenido, en el caso particular de la UNED, el curso es de forma general.

\section{RESULTADOS Y DISCUSIÓN}

Los principales resultados encontrados a la hora de analizar los programas de estudios demuestran que no existen cursos especializados en documentación audiovisual dentro de la malla curricular de las Universidades que imparten la carrera de Bibliotecología y archivística. Sin embargo, al menos en la formación profesional logran tener una noción básica, tal como lo expresa Royan y Cremer "De la misma manera que es importante que un bibliotecario [y un archivista] tenga conocimientos sobre literatura cuando trabaja con libros, [o documentos] es importante tener conocimientos sobre películas, pintura, música, etc., cuando se trabaja con material audiovisual" (2004, p.4).

Otro aspecto importante de señalar es que al no contar con una formación en documentación audiovisual, tampoco existen profesionales especialistas, razón por la cual se carece de profesores especialistas. Tanto los estudiantes como el mismo profesor de las dos disciplinas poseen una misma formación.

El hecho de no contar con personal especializado repercute de forma directa en las unidades de información, principalmente en aquellas cadenas televisivas que tienen sus propios acervos y que en muchos de los casos son atendidas por otros especialistas, que no cuentan con la formación bibliotecólogica o archivistica.

"El desarrollo profesional continuo es especialmente necesario para los especialistas en materiales multimedia, debido a los rápidos cambios de técnicas y productos. Este desarrollo debe ofrecerse en los programas de las asociaciones profesionales, escuelas de biblioteconomía y ciencias de la información, y otras organizaciones relacionadas. Tales programas pueden organizarse a escala internacional, nacional, regional, o local".

Finalmente, es importante recalcar el papel de las universidades formadoras de los bibliotecólogos y archivistas en realizar una revisión de sus planes de estudio que como bien indica la Mta. Escalona Ríos. (2008) “[...] cuando la información se convierte en un elemento dinámico alrededor del cual gira la economía y producción de un país, tanto el perfil como los planes tienen que cambiar" (p.13). 


\section{CONCLUSIONES}

Es necesario brindar una formación o especialidad a los estudiantes que actualmente estudian las carreras de Documentación, Ciencias de la información y Archivistica donde se asegure las técnicas y métodos de trabajo, servicios y productos propios de la especialización en documentación audiovisual.

A su vez, las escuelas de formación de bibliotecólogos y archivistas deben de ofrecer cursos de especialización y actualización, profesional a los profesionales responsables de trabajar con acervos audiovisuales.

Finalmente, resulta urgente abrir la especialidad para poder establecer líneas de investigación en las escuelas, que genere conocimiento en el desarrollo de estas dos disciplinas.

\section{REFERENCIAS BIBLIOGRÁFICAS}

AFURA, Angeles . "El departamento de documentación de la Ser, ante el reto digital”. Cuadernos de Documentación Multimedia. 1998. vol. 6-7, p. 1-4.

BARANDA DEL CAMPO, Cristina. "El espacio común entre dos disciplinas: Periodismo y Documentación". Cuadernos de Documentación Multimedia. 2013. ISSN1575-9733.

BUSTAMANTE MORA, Cynthia, CASCANTE ARDÓN, Priscila,; CORDERO MÉLENDEZ, Flory; RODRÍGUEZ VINDAS, Dana; SAAVEDRA ARIAS, Grettel y ZÚÑIGA VILLALOBOS, Florybeth . "El mercado laboral de las personas graduadas de la carrera de Bachillerato en Bibliotecología con énfasis en Ciencias de la Información de la Universidad de Costa Rica que laboran en la Gran Área Metropolitana”. (Tesis). San José : Universidad de Costa Rica. Escuela de Bibliotecología y Ciencias de la Información. 2014.

CALDERA SERRANO, Jorge; ARRANZ-ESCACHA, Pilar. "Documentación audiovisual en televisión”. Colección El profesional de la información. 13. 2012. Barcelona: Editorial UOC.

CALDERA SERRANO, Jorge y ZAPICO ALONSO, Felipe. . "Procedencia de información audiovisual en las empresas televisivas". Hipertext.net, $2003 . \quad$ no.1. http://www.upf.edu/hipertextnet/numero-1/empresas tv.html.

CALDERA SERRANO, Jorge; ZAPICO ALONSO, Felipe. "La fórmula de Lasswell como método para implementar bases de datos documentales en los medios audiovisuales". Investigación Bibliotecológica. 2004. vol.8, num. 37, p.110-131.

CALDERA SERRANO, Jorge; SÁNCHEZ JIMENEZ, Rodrigo . "Desarrollo y aplicación de una ontología para el control y recuperación de información onomástica en televisión”. El Profesional de la Información. 2007. vol. 17, núm. 1, pp. 86-91.

CASANOVA, Fernando. . Formación profesional y relaciones laborales. Montevideo: CINTERFOR $\begin{array}{llll}\text { (Sindicatos } \quad \text { Formación, } & 2003 .\end{array}$ www.oei.es/etp/formacion_profesional_relaciones_laborales.pdf. 
ECHEVARRÍA SOLÍS, Ana Lorena y RIVAS FERNÁNDEZ, José Bernal. . "La apertura de la Licenciatura en Archivistica en la Universidad de Costa Rica: Proceso metodológico”. Información, $\begin{array}{llllll}\text { Cultura } y & \text { Sociedad. } 2006 . & \text { num. } & 14 . & \text { ISSN }\end{array}$ http://www.scielo.org.ar/scielo.php?script=sci_arttext\&pid=S1851-17402006000100004

ECHEVARRÍA SOLÍS, Ana Lorena [2002]. La carrera de Archivística en la Universidad de Costa Rica. www.archivonacional.go.cr/memorias/2002/04.doc

ESCALONA RÍOS, Lina. Hacia el establecimiento del perfil del bibliotecólogo a nivel de Iberoamérica. México: UNAM. CUIB. 2008.

LÓPEZ DE QUINTANA, Eugenio. “Documentación en televisión”. Manual de documentación informativa. Madrid: Cátedra, 2000. p. 83-181.

HIDALGO GOYANES, Paloma. "La documentación audiovisual de las televisiones. La problemática actual y el reto de la digitalización”. Documentación de las Ciencias de la Información, 2005. 28, p.159-171

LÓPEZ YEPES, Alfonso. Manual de documentación audiovisual. Pamplona: Ediciones de la Universidad de Navarra. 1992.

MICÓ SANZ, Josep Lluís; MASIP MASIP, Pere y GARCÍA AVILÉS, José Alberto. "Redefinición del perfil y funciones del documentalista en las redacciones digitales de medios españoles". IX Jornadas de Gestión de la Información: Informar y difundir servicios documentales y comunicación. Madrid: SEDIC, 2007. p. 105-119.

MARCOS RECIO, Juan Carlos. “El Documentalista de Información Electrónica”. Documentación de las Ciencias de la Información. 1996. num. 19. ISSN 0210-4210. p. 193-210.

MÚÑERA TORRES, María Teresa. "Formación del profesional en bibliotecología y archivista". Revista Códice. 2010. Vol.6, num. 1. p. 81-87.

PÉREZ AGUERA, José Ramón; SÁNCHEZ JIMENEZ, Rodrigo; CALDERA SERRANO, Jorge . "Adaptación de tecnologías stream y xml a centros de documentación en televisión". Revista Española de Documentación Científica. 2004. vol. 27, núm. 4, p. 441-454.

PIRELA MORILLO, Johann E. Mercados laborales y perfiles por competencias del profesional de la información. Algunos estudios iberoamericanos. Formación, Investigación y Mercado Laboral en Información y Documentación en España y Portugal / José Antonio Frias y Crispulo Travieso (eds). Salamanca : Ediciones Universidad de Salamanca. 2008. ISBN978-7481-654-9. p. 527. 
RODRÍGUEZ RESÉNDIZ, Perla Olivia. "Documentación audiovisual y nuevas tecnologías de información en el ámbito educativo". Contribuciones desde Coopatec. (jul.-dic. 2001). num. 1. p. 140-147. ISSN 1870-0365.

RODRIGUEZ SALAS, Karla. El profesional en bibliotecología y documentación: habilidades y competencias. 2008. http://goo.gl/0jpRtK

ROYAN, B y CREMER, M. (2004). Directrices para materiales audiovisuales y multimedia en bibliotecas $y$ otras instituciones. Traducido por Lourdes Feria Basurto y Luis Felipe Medina Alvarez. IFLA.Headquaters. (IFLA Professional Reports, Nr. 84).

TEJADA ARTIGAS, Carlos Miguel. Perfil, motivaciones y expectativas de los alumnos de la Diplomatura en Biblioteconomía y Documentación: un acercamiento a través de una encuesta de la Facultad de Ciencias de la Documentación de la Universidad Complutense de Madrid (curso 2006-2007). Formación, Investigaciṕon y Mercado Laboral en Información y Documentación en España y Portugal / José Antonio Frias y Crispulo Travieso (eds). Salamanca : Ediciones Universidad de Salamanca. 2008. ISBN978-7481-654-9. p.591.

UNIVERSIDAD DE COSTA RICA. Programa del curso BI-2006 Medios de Comunicación. San José, Costa Rica, [pdf] 2015.

UNIVERSIDAD DE COSTA RICA. Programa del curso BI-3005 Desarrollo de Colecciónes. San José, Costa Rica, [pdf] 2015.

UNIVERSIDAD DE COSTA RICA. Programa del curso BI-4006 Centros de Información Especializadas. San José, Costa Rica, [pdf] 2015.

UNIVERSIDAD DE COSTA RICA. Programa del curso HA-5005 Gestión de Documentos II. San José, Costa Rica, [pdf] 2015.

UNIVERSIDAD DE COSTA RICA. Programa del curso HA-5006 Tecnologías de la Información y Comunicación Aplicada. San José, Costa Rica, [pdf] 2015.

UNIVERSIDAD DE COSTA RICA. Programa del curso HA5018 Sistemas de Información Archivistica. San José, Costa Rica, [pdf] 2015.

UNIVERSIDAD NACIONAL DE COSTA RICA. Programa del curso BGE-212 Aplicación de los multimedios. Heredia, Costa Rica, [pdf] 2015.

UNIVERSIDAD NACIONAL DE COSTA RICA. Programa del curso BGE-415 Diseño de interfaces gŕaficas. Heredia, Costa Rica, [pdf] 2015.

UNIVERSIDAD NACIONAL DE COSTA RICA. Programa del curso BGE-205 Documentación. Heredia, Costa Rica ,[pdf] 2015. 
UNIVERSIDAD NACIONAL DE COSTA RICA. Programa del curso BGE-212 Aplicación de los multimedios. Heredia, Costa Rica, [pdf] 2015.

UNIVERSIDAD NACIONAL DE COSTA RICA. Programa del curso BGE-415 Diseño de interfaces gŕaficas. Heredia, Costa Rica, [pdf] 2015.

UNIVERSIDAD NACIONAL A DISTANCIA. Programa del curso 5082 Lenguajes documentales y sistemas de gestión documental. San José, Costa Rica ,[pdf] 2015.

UNIVERSIDAD NACIONAL A DISTANCIA. Programa del curso 5305 Innovaciones en las Tecnologías de la Información Comunicación. San José, Costa Rica ,[pdf] 2015.

VALLE, Felix del. Manual de documentación fotográfica. Madrid: Síntesis. 1999.

VALLEJO SIERRA, Ruth Elena. "La formación profesional del bibliotecólogos y archivistas: integrada con énfasis o independiente”. World Library and Information Congress. 77 IFLA. General Conference. Meeting 110-LIS Education in Developming Countries Special Interest Group with Latin America and the Carribbean. 2011. conference.ifla.org/past-wlic/2011/110sierra-es.pdf. 